\title{
Locally compact groups which are just not compact
}

\author{
Francesco Russo \\ Department of Mathematics, University of Naples Federico II, \\ via Cinthia80126, Naples, Italy; E-mail: francesco.russo@dma.unina.it
}

\begin{abstract}
A Just-Non-Compact group, or briefly a JNC group, is a Hausdorff topological group which is not a compact group but all of whose proper Hausdorff quotients are compact groups. Intuitively, it is clear that these groups are rich in compact quotients. Locally compact JNC groups are largely described in the present paper.
\end{abstract}

Keywords Just-Non-Compact groups, Lie groups, compact groups.

MR(2000) Subject Classification 22C05; 20E22; 20E34

\section{Introduction}

A long line of research is devoted to the investigation of the structure of a group which does not satisfy a prescribed property but all of whose proper quotient groups satisfy it. Literature can be found in [1, Chapters 6 and 11], in [14] and recently in $[10,11,12,13]$. The interest for these groups is so deep that many authors investigated them and we cannot find a uniform terminology in the topic. For instance, H. Schunk called these groups the groups of boundary $\mathfrak{X}$, where $\mathfrak{X}$ is a prescribed property (see [1, Chapter 3]). The same groups are called Just-Non- $\mathfrak{X}$ groups by many authors, as we may note in the corresponding literature. See $[10,11,12,13,14]$ for details. Thus, it is common that a same result can be found independently by another author in a different terminology. Looking at the literature we may note that arguments and methods deal with generalizations of a famous Splitting's Theorem of I. Schur and H. Zassenhaus (see [1, Chapter A, §11, 11.3]). We will note these analogies in Theorems 2.5, 2.6 and 2.7 of the next section. They are the main results of the present paper.

We saw in [10] that Just-Non- $\mathfrak{X}$ groups can be described in a satisfactory way in the context of topological groups. We concentrated in [10] on compact groups and on the property to be a Lie group. In this paper we consider a slightly different situation and apply some of the methods developed in [10]. Most of our

Received XXX, XXX, Revised XXX, Accepted XXX, XXX

Supported by GNSAGA of INDAM, via Madonna del Piano, Sesto Fiorentino (FI), Italy 
notation and terminology is standard and can be found in $[2,3,5,6,7,15]$, which are classic references for topological groups.

\section{Main results}

A Just-Non-Compact group, or briefly a JNC group, is a Hausdorff topological group which is not a compact group but all of whose proper Hausdorff quotients are compact groups. Note that a normal subgroup $N$ of a topological group $G$ is closed if and only if the quotient group $G / N$ is Hausdorff. All noncompact simple groups (that is, groups without proper nonsingleton closed normal subgroups) are JNC groups by default. So one should concentrate on the nonsimple groups in the class. From now we assume the Hausdorff separation for a topological group.

If $G$ is discrete, then $G$ is a JNC group if and only if $G$ is an infinite group all of whose proper quotients are finite groups: this is another topic of interest to the algebraic theory of groups, and we assume from here on out that a JNC group is a nondiscrete nonsimple topological group.

Let $G$ be a topological group and let $\mathcal{N}(G)$ denote the set of all normal closed subgroups of $G$ such that $N \in \mathcal{N}(G)$ if and only if $G / N$ is a compact Lie group. Then $G \in \mathcal{N}(G)$; further $\{1\} \in \mathcal{N}(G)$ if and only if $G$ is a compact Lie group. If $\mathcal{N}(G)$ contains arbitrarily small members, then $\mathcal{N}(G)$ is a filter basis. For instance, see [7, pp.148-149]. It seems opportune to point out that here and in the following a Lie group is a finite dimensional real Lie group. Consider

$$
\mathcal{C}(G)=\{N: N \text { is a closed normal subgroups of } G \text { with }\{1\} \neq N \neq G\}
$$

and the closed normal subgroup $D_{G}=\bigcap \mathcal{C}(G)$ of $G$. Note that

$$
\mathcal{N}(G)=\{N \in \mathcal{C}(G) \cup\{\{1\}, G\}: G / N \text { is a Lie group }\} .
$$

We say that $G$ is residually compact if $D_{G}=\{1\}$. In any case $G / D_{G}$ is residually compact. If $G$ is a JNC group, then there are just two possibilities: either $D_{G}=\{1\}$ or $D_{G} \in \mathcal{C}(G)$. The first case occurs for instance for $G=\mathbb{R}^{n}$. Since an extension of a compact group by a compact group is a compact group, $N$ is not compact for each $N \in \mathcal{C}(G)$, then in the second case $D_{G}$ is not compact. In particular, the following lemma is clear.

Lemma 2.1. If $G$ is a JNC group, then all members $N \in \mathcal{C}(G)$ are noncompact groups.

This motives to look for the normal subgroups of a topological group. Recall that a topological group $G$ is called almost connected if $G / G_{0}$ is a compact group, 
where $G_{0}$ denotes the identity component of $G$. Note that $G_{0} \in \mathcal{C}(G) \cup\{\{1\}, G\}$. So we have the following lemma.

Lemma 2.2. A JNC group $G$ is either totally disconnected or almost connected with noncompact identity component.

Proof. If $G_{0}=\{1\}$, then $G$ is totally disconnected. If $G_{0}=G$, then $G$ is connected and thus almost connected. If $G_{0} \in \mathcal{N}(G)$, then $G_{0}$ is noncompact by Lemma 2.1 and so $G / G_{0}$ is a compact group.

We know that all locally compact almost connected groups are pro-Lie groups by Yamabe's Theorem. See for details $[16,17]$. This famous result proves an old conjecture of A. M. Gleason in [4] and can be found in different formulations in $[2,3,5,6,7,15]$. A classical source for a proof is [9]. We will refer to [7] for pro-Lie groups and to [15] for locally compact groups.

Theorem 2.3. A JNC pro-Lie group $G$ is a Lie group and is a product $G=G_{0} F$ where $F$ is a finite group.

Proof. Assume that $G$ is not a Lie group and $\{1\} \neq N \in \mathcal{N}(G)$. Then $\mathcal{N}(G)$ is a filterbasis $\mathcal{F}$ of normal subgroups such that $G / N$ is a compact Lie group and $G=\lim _{N \in \mathcal{F}} G / N$ (see [7, Proposition 3.27]). Now each $G / N$ is a compact group, since $G$ is a JNC group. Hence $G$ is a compact group as projective limit of compact groups. This is a contradiction and so $G$ is a Lie group.

By Lemma 2.2, $G$ is either totally disconnected or almost connected. Since a totally disconnected Lie group is discrete, this case is not allowed by the definition of a JNC group. Hence $G$ is an almost connected group which means that $G$ has only finitely many connected components. By Dong Hoon Lee's Supplement Theorem [8, Theorem 2.13], there is a finite group $F$ such that $G=G_{0} F$ and the result follows.

Now we recall some basic notions which can be found in $[2,3,5,6,7,15]$. Every pro-Lie group $G$ has a unique largest compact normal subgroup $C(G)$ (see for instance [7, Theorem 104]). We recall that the nilradical $N(G)$ of a Lie group $G$ is the largest connected nilpotent normal subgroup of $G$ (see for instance [7, Definition 10.40, Theorem 10.42]). Then we may deduce the following lemma.

Lemma 2.4. Assume that $G$ is a pro-Lie group. If $G$ is a JNC group, then $N(G)$ is either a singleton or a vector group. 
Proof. Assume that $N(G) \neq\{1\}$. Since $G$ is a pro-Lie group and also a JNC group, $C(G)=\{1\}$. The closed commutator subgroup $\overline{[N(G), N(G)]}$ is characteristic in $G$. If $N(G)$ is nonabelian, then $N(G) / \overline{[N(G), N(G)]}$ is compactfree, contrary to the fact that $G$ is a JNC group. Hence $N(G)$ is abelian and, since $C(G)=\{1\}, N(G)$ is a vector group. Note that $N(G)$ is isomorphic to $\mathbb{R}^{n}$ for some $n \geq 1$.

Before of the next result, we should recall two usual notions which can be found in $[2,3,5,6,7,15]$. We may identify a compact Lie group $K$ with a closed subgroup of the orthogonal group $\mathrm{O}(n)$ of $\mathbb{R}^{n}$ with respect to a suitable positive definite inner product on $\mathbb{R}^{n}$. When this happens, we say that $K$ acts (or operates) ef fectively on $\mathbb{R}^{n}$. On another hand, the notion of irreducible action is well known in the representation theory of compact groups.

Theorem 2.5. Assume that $G$ is a pro-Lie group with $N(G) \neq\{1\}$. If $G$ is a JNC group, then there exists an integer $n \geq 1$ such that $G \simeq \mathbb{R}^{n} \rtimes K$, where $K$ is a compact Lie group operating irreducibly and effectively on $\mathbb{R}^{n}$.

Proof. From Theorem 2.3 we may assume that $G$ is a Lie group. From Lemma 2.4 , we know that there exists an integer $n \geq 1$ such that $N(G) \simeq \mathbb{R}^{n}$. By the definition of JNC group, $G / N(G)$ is a compact group. Then we may apply the Vector Group Splitting Theorem [7, Theorem 11.15] so that $G \simeq \mathbb{R}^{n} \rtimes K$ for a maximal compact subgroup $K$ of $G$. Since the representations of a compact group on a finite dimensional vector space are completely reducible, a $K$-invariant nonsingleton proper vector subgroup $V$ of $N(G)$ would be in $\mathcal{N}(G)$. But, $G / V$ would contain the noncompact subgroup $N(G) / V$. So $K$ acts irreducibly on $N(G)$. The set of the elements of $K$ fixing $N(G)$ elementwise is $D:=K \cap$ $C_{G}(N(G))$, where $C_{G}(N(G))$ is the centralizer of $N(G)$ in $G$. The normalizer of $D$ in $G$ contains $K$ and $N(G)$. Thus $D$ is normal in $G$ and fails to meet $N(G)$, whence $G / D$ fails to be compact. Hence $D=\{1\}$, that is, $K$ acts effectively on $N(G)$. The result follows.

As already noted, in Theorem $2.5 \mathrm{~K}$ may be identified with a closed subgroup of the orthogonal group $\mathrm{O}(n)$ of $\mathbb{R}^{n}$ with respect to a suitable positive definite inner product on $\mathbb{R}^{n}$. Conversely, every closed subgroup $K$ of $\mathrm{O}(n)$, acting irreducibly on $\mathbb{R}^{n}$ give arise to a JNC group $\mathbb{R}^{n} \rtimes K$.

Note that the case in which $K$ is singleton is not excluded; the singleton group acts irreducibly and effectively on $\mathbb{R}$, and so $G=\mathbb{R}$ is included in the class of JNC groups, classified in Theorem 2.5. 
Theorem 2.6. Assume that $G$ is a pro-Lie group with $N(G)=\{1\}$. If $G$ is a JNC group, then there exists an integer $n \geq 1$ such that $G \simeq S^{n} F$, where $S$ is a centerfree simple connected noncompact Lie group, $S^{n} \simeq G_{0}$ and $F$ is a finite group acting transitively on $\{1, \ldots, n\}$.

Proof. By Theorem 2.3 we may assume that $G$ is a Lie group. Now $G_{0}$ is reductive. Indeed $G_{0}$ is semisimple, since in a reductive connected Lie group, the identity component of the center agrees with $N(G)$. We observed that $G$ does not have compact normal subgroups; the maximal compact normal subgroup of a semisimple Lie group is characteristic hence normal in $G$, and this is impossible. Suppose $Z\left(G_{0}\right) \neq\{1\}$; then $Z\left(G_{0}\right) \in \mathcal{C}(G)$ and thus $G / Z\left(G_{0}\right)$ is compact, since $G$ is a JNC group. We conclude that $G_{0} / Z\left(G_{0}\right)$ is a compact group. This contradicts the fact that $G_{0}$ is semisimple without compact factors. Thus $G_{0}$ is centerfree semisimple and it is a product $S_{1} \times \ldots \times S_{n}$ of adjoint noncompact simple Lie groups. Any nondegenerate proper characteristic subgroup $C$ of $G_{0}$ would be normal in $G$ and those belong to $\mathcal{C}(G)$ whence $G / C$ and thus $G_{0} / C$ would be compact, contradicting the absence of compact factors. Thus $S_{1}, \ldots, S_{n}$ must be isomorphic and we may write $G_{0}=S^{n}$ with a centerfree noncompact simple Lie group $S$. Now we invoke Dong Hoon Lee's Supplement Theorem [8, Theorem 2.13] and conclude that there is a finite subgroup $F$ of $G$ such that $G=G_{0} F$. The group $F$ acts on $S^{n}$ by permuting the factors transitively in order that no normal subgroups of $G$ arise. The result follows.

In essence, the results of the present section say that we know explicitly what the locally compact JNC groups are, if they are not totally disconnected. In the direction of totally disconnected groups, we know only that locally compact JNC groups are not prodiscrete (see [7, p.15]). The examples we constructed above can be mimicked to some extent by using the irreducible and faithful representations of certain compact groups over finite dimensional vector spaces over nonarchimedean locally compact fields. However, this seems to be a wide field. We want to finish the present paper with another formulation of the circumstances in Theorems 2.5 and 2.6.

Theorem 2.7. Let $G$ be a locally compact but not totally disconnected JNC group. Then $G$ is a Lie group of one of the following two types:

(i) $G \simeq \mathbb{R}^{n} \rtimes_{\pi} K$ for an irreducible and faithful representation of a compact Lie group $\pi: K \rightarrow \mathrm{GL}(n, \mathbb{R})$.

(ii) There is a simple centerfree noncompact Lie group $S$ and a finite subgroup $F$ of $G$ such that $G_{0} \simeq S^{n}$ and $G=G_{0} F$ and $F$ acts transitively on $\{1, \ldots, n\}$. 
Proof. If $G$ is a locally compact but not totally disconnected JNC group, then it is almost connected by Lemma 2.2. Then $G$ is a pro-Lie group by Yamabe's Theorem. But then it is a Lie group by Theorem 2.3. Hence Theorems 2.5 and 2.6 taken together prove the theorem.

The absence of $K$-invariant vector subspaces of $\mathbb{R}^{n}$ in Theorem 2.5 and Theorem 2.7 (i) need not mean the absence of $K$-invariant nontrivial subgroups of $\mathbb{R}^{n}$. In the case of the dihedral group $\mathbb{R} \rtimes\{1,-1\}$, the two element group acts irreducibly and effectively on $\mathbb{R}$, but each cyclic subgroup $\mathbb{Z} r$ of $\mathbb{R}$ is invariant. In this situation we have $D_{G}=\{1\}$. Some other examples are listed below.

Examples 2.8. The following groups all are realizable.

(1). Let $\mathbb{K}$ be a nondiscrete locally compact field and $\pi: K \rightarrow \operatorname{GL}(n, \mathbb{K})$ be an irreducible representation of a compact group $K$. Then $G=\mathbb{K}^{n} \rtimes K$ is a JNC group (indeed $D_{G}=\mathbb{K}^{n} \times\{1\}$ ).

(2). Let $L$ be a simple (and indeed centerfree) Lie group over a nondiscrete locally compact field $\mathbb{K}$. Let $F$ be a finite group acting transitively on $S=\{1, \ldots, n\}$. Then we obtain a representation $\pi: F \rightarrow \operatorname{Aut}\left(L^{S}\right)$ via actions by permuting the factors. Then $G=L^{S} \rtimes_{\pi} F$ is a JNC group (with $D_{G}=L^{S} \times\{1\}$ ).

All of these examples are totally disconnected except in the cases that $\mathbb{K}=\mathbb{R}$ or $\mathbb{K}=\mathbb{C}$. In these cases the examples are Lie groups.

Acknowledgements. I am grateful to the referee for many significant suggestions and especially for the improvement of Theorems 2.5 and 2.6 in form of Theorem 2.7.

\section{References}

[1] Doerk, K. and Hawkes, T., Finite Soluble Groups, de Gruyter, Berlin, 1992

[2] Bourbaki, N., Lie Groups and Lie Algebras: Chapters 4-6 (Elements of Mathematics), Springer, 2002

[3] Bourbaki, N., Lie Groups and Lie Algebras: Chapters 7-9 (Elements of Mathematics), Springer, 2004

[4] Gleason, A. M.: The Structure of Locally Compact Groups. Duke Math. J. 18, 85-104 (1951)

[5] Hewitt, E. and Ross, K. A., Abstract Harmonic Analysis, Vol.I, Springer, New York, 1963

[6] Hofmann, K. H. and Morris, S. A., The Structure of Compact Groups, de Gruyter, Berlin 2006

[7] Hofmann, K. H. and Morris, S. A., The Lie Theory of Connected Pro-Lie Groups, European 
Mathematical Society, Zürich 2007

[8] Lee, D. H.: Supplements for the identity component in locally compact groups, Math. Z. 104, 28-49 (1968)

[9] Montgomery, S. and Zippin, L., Topological Transformation Groups, Interscience Publishers, New York, 1955

[10] Russo, F.: On Compact Just-Non-Lie Groups. J. Lie Theory 17, 625-632 (2007)

[11] Russo, F.: A Note on Just-Non- $\mathfrak{X}$ groups. Int. J. Algebra 2, 277-290 (2008)

[12] Russo, F.: Some Groups Whose Proper Quotients Have Soluble Minimax Conjugacy Classes. Int. J. Algebra 2, 291-299 (2008)

[13] Russo, F.: Groups With Many Quotients Which Are PC-groups. Int. J. Contemp. Math. Sci. 3, 1041-1058 (2008)

[14] du Sautoy, M., Segal, D. and Shalev, A., New Horizons in Pro-p-groups, Progress in Mathematics 184, Birkhäuser, Boston, 2000

[15] Stroppel, M., Locally Compact Groups, European Mathematical Society, Zürich, 2006

[16] Yamabe, H.: Generalization of a theorem of Gleason. Ann. of Math. 58, 48-54 (1953)

[17] Yamabe, H.: On the conjecture of Iwasawa and Gleason. Ann. of Math. 58, 351-365 (1953) 